\title{
EL AMBIENTE EN UN AULA DEL CICLO DE TRANSICIÓN
}

\section{Ana Polanco Hernández ${ }^{1}$}

Resumen: El artículo en su inicio expone la conceptualización que distintos pedagogos han asignado a través de la historia al ambiente físico en el jardín infantil. Luego se plantean características de tamaño, iluminación, decoración de paredes, mobiliario y la división por áreas de trabajo que puede poseer un aula del ciclo de transición, Se hace una descripción de cada área y el material didáctico que es recomendable que posean. cada una de ellas.

Se propone las características del mobiliario, el cual debe adaptarse a no solo a las necesidades e intereses de los niños y niñas, si no también debe ser acorde con la metodología que se trabaje. Por último se comenta el papel de los materiales.

Palabras clave: EDUCACIÓN PREESCOLAR/ AMBIENTE DE AULA/ AREAS DE TRABAJO/ MATERIAL DIDÁCTICO/

Abstracts: The article in its beginning exposes the conceptualization that different instructors have assigned through history to the physical atmosphere in the infantile garden. Soon characteristics of size, illumination, decoration of walls, furniture consider and the division by work areas that can have a classroom of the cycle of transition, becomes a description of each area and the didactic material that is recommendable that has each one of them. One sets out the characteristics of the furniture, which must adapt to nonsingle to the necessities and interests of the children and children, if not also it must be agreed with the methodology that works. Finally the paper of the materials comments.

Key words: PREESCHOOL EDUCATION/ CLASSROOM ENVIRONMENT/ WORK'S AREAS/ DIDACTIC MATERIAL /

\section{INTRODUCCIÓN}

El ambiente en el jardín infantil es de vital importancia, dado que la mayoría de las situaciones de aprendizaje que se dan durante la rutina diaria, suceden dentro del salón de clase.

Es relevante considerar el espacio, la distribución del mobiliario, puesto que estos elementos contribuyen a las relaciones interpersonales que se dan dentro del aula, favorecen la construcción del conocimiento y colaboran con el éxito de las situaciones de aprendizaje. Al respecto, Hohmann, et, al. (1984) exponen que "el espacio es importante porque afecta todo lo que hace el niño, influye en su actividad, en su trabajo, en sus elecciones, en la forma de utilizar los materiales así como en las relaciones con los demás."

\footnotetext{
${ }^{1}$ Master en Planificación Curricular, Licenciada en Educación Preescolar, Bachiller en Educación Preescolar, todos los títulos de la Universidad de Costa Rica. Profesora de la Escuela Formación Docente, Sección de Preescolar. Coordinadora Proyecto de Acción Social "Apoyo Docente a Instituciones Públicas de Educación Inicial".
}

Correo electrónico: apolanco@,costarricense.cr

Artículo recibido: 21 de abril, 2004

Aprobado: 21 de junio, 2004 
La organización del espacio de un aula, la disposición del mobiliario y de los materiales motiva a los niños a moverse en una u otra dirección, jugar en un determinado lugar o cambiarse a otro, escoger los materiales, comunicarse, desplazarse, cambiar de materiales o no, y otros. (Iglesias, 1996)

Es fundamental que los docentes de educación preescolar estén conscientes de la importancia de los espacios al planificar su trabajo, y se preocupen por la manera en que son ordenados, equiparlos y enriquecerlos para que se conviertan en factores estimulantes de la actividad.

Este artículo pretende destacar la manera en que el ambiente influye en el aprendizaje, las características generales de un aula de educación preescolar del Ciclo de Transición, la división de los espacios, las áreas que puede contener, así como opciones de material didáctico con que es posible equipar cada una de ellas.

\section{Ambiente en un aula del Ciclo de Transición}

A menudo se confunde los términos espacio físico y ambiente físico, probablemente por encontrarse interrelacionados; no obstante, son diferentes, dado que el espacio físico se refiere al local donde se realizan las actividades, el cual se caracteriza, por material, por el mobiliario, la decoración y los objetos; mientras que el ambiente, es el conjunto del espacio físico y las relaciones que se establecen en él; como por ejemplo, los afectos y las interrelaciones entre las niñas y los niños y el docente. (Iglesias, 1996)

Cuando se hace referencia al ambiente de aula, éste es posible visualizarlo desde dos puntos de vista, el afectivo o el físico, no obstante para efectos de este artículo se conceptualizará el ambiente físico, el cual se define como el conjunto de relaciones interpersonales que se dan en el aula, y el espacio físico en el que se lleva a cabo la labor educativa. Al respecto, Iglesias, (1996) define el ambiente como "un todo indisociado de objetos, olores, formas, colores, sonidos y personas que habitan y se relacionan en un determinado marco físico que lo contiene todo, y al mismo tiempo, es contenido por todos estos elementos que laten dentro de él como si tuvieran vida". 
Por esto, el mobiliario del aula, su distribución, las paredes, los murales, los materiales, el modo en que estén organizados y la decoración, indican el tipo de actividades que se realizan, las relaciones que se dan, así como los intereses de los niños.

La educación preescolar, desde su inicio, ha resaltado el valor del espacio y del ambiente físico como elementos fundamentales del quehacer educativo.

Froebel resaltaba el espacio exterior como facilitador, pues permite el desarrollo de actividades variadas y espontáneas. Desde su perspectiva, con respecto al espacio interior, lo más relevante era que éste sea amplio y ventilado para que el niño pueda realizar actividades variadas y desarrollar sus potencialidades. El tamaño del mobiliario debe ser proporcional a la estatura de los niños. (Peralta, 1996)

Rosa Agazzi y Carolina Agazzi, educadoras italianas de finales del siglo XIX, con respecto del ambiente consideran la higiene como elemento esencial en un centro infantil y el salón de clase tuviera buena ventilación, iluminación y calefacción. Plantean la creación de un "museo didáctico" dentro del aula, compuesto por los objetos que los niños traían en sus bolsillos, con esto se introducen en el jardín infantil materiales de deshecho como un recurso válido dentro del currículo preescolar. También, es importante resaltar que las hermanas Agazzi fueron las primeras en aportar el uso de contraseñas o distintivos en cada material. (Peralta, 1996)

María Montessori, propuso un ambiente estructurado que diera posibilidades de acción y elección del niño, en donde el material del aula estaba determinado por los objetivos. Para ella, es de suma importancia el material que se proporciona, el cual debe ser liviano, para que el niño pueda transportarlo y de esta forma, favorecer la libertad, la autonomía y la independencia. El mobiliario del aula posee características especiales en sus formas y colores. El ambiente externo debe favorecer en el niño el contacto con la naturaleza. (Montessori, 1939)

Para Ovideo Decroly, es importante que el niño tenga contacto con la naturaleza, por esto el jardín de infantes debe poseer zonas verdes. El aula debe ser tipo "taller" con decoraciones elaboradas por los niños y contar con una sala comedor. (Peralta, 1996) 
Con el transcurso del tiempo, la educación preescolar ha sufrido cambios producto de nuevas investigaciones, no obstante, aún mantiene algunas características que plantearon sus propulsores.

Por otro lado, también, investigadores como Piaget y Vigotski, hacen referencia a la importancia del ambiente para la adquisición y construcción de nuevos conocimientos en el niño.

Piaget plantea tres tipos de conocimiento: el físico, el lógico matemático y el socioconvencional. La fuente principal del primero está parcialmente en la realidad externa de los objetos. Este conocimiento se adquiere cuando el niño física y mentalmente actúa sobre los objetos y descubre cómo reaccionan. El segundo, tiene su fuente principal en la mente del niño al leer la información externa de la realidad. Por último, el socio-convencional se refiere, por ejemplo, a las normas sociales que el niño adquiere en el medio en el cual se desarrolla (Zamora, 1998).

Piaget afirmó que los niños pequeños conocen los objetos al actuar sobre ellos y al conocer sus reacciones, esta información sensorial se convierte en conocimiento únicamente en el contexto de la acción del niño sobre los objetos. (Zamora, 1998)

Vila Ignasi (1997) plantea que desde el punto de vista de Vigotski, la forma como se organizan socialmente los espacios, los materiales y las actividades, es importante en la educación infantil, a partir del contexto sociocultural en el que se desenvuelve el niño.

Así, el ambiente físico es de vital importancia en el proceso educativo, al respecto García, et, al. (1992), propone que el aprendizaje del niño se da mediante la construcción de conocimientos generados por medio de interacciones con otros niños, con el maestro y con los recursos; de esta forma el pequeño explora, experimenta y construye.

El espacio, visualizado como contenido curricular, se ha dado como un proceso de apropiación por parte de los docentes en diferentes etapas.

Inicialmente, el espacio funcionaba como una variable ante la cual nada podía hacer el maestro. Esta impulsividad se daba por cultura curricular. No se consideraba que el espacio 
pudiera ser manejado, éste era concebido como el lugar donde se enseñaba. Los profesores se adaptaban al espacio de la mejor forma que dispusieran.

Luego, el espacio se convirtió en un componente instrumental que el docente podía cambiar de acuerdo con el trabajo que deseaba realizar, entonces el maestro sabía que podía tomar decisiones sobre este componente curricular denominado "espacio".

Finalmente, el espacio se convierte en una variable básica, no es ya solamente en el espacio en el que se trabaja o el elemento facilitador, si no que constituye un factor de aprendizaje. Así, actualmente, las maestras de educación preescolar al planificar su trabajo toman decisiones en relación con los espacios, por ejemplo: distribución, materiales y recursos, con el objetivo de que se conviertan en factores estimulantes de la actividad y que favorezcan el acceso de los niños.

Bajo esta última concepción la organización del espacio, es un aspecto curricular que el docente considera importante, dado que lo visualiza como un cúmulo de recursos de aprendizaje y de desarrollo personal.

La organización que se le dé a un salón de clase es tan importante que la distribución del mismo indica los planteamientos pedagógicos, el modo en el que se entiende el proceso enseñanza y aprendizaje, así como las creencias educativas de los adultos responsables.

\section{EL AULA}

El ambiente del salón de clase es importante, dado que este es el sitio en que se llevan a cabo la mayoría de las actividades que se realizan; por esto, es fundamental que este favorezca la estimulación en las áreas del desarrollo integral (socioafectiva, sicomotora y cognitiva-lingüística) del educando.

El tamaño del aula debe ser lo suficientemente grande como para que los niños puedan desplazarse libre y cómodamente al trabajar, así como poseer la capacidad de albergar el mobiliario y material necesario para favorecer la labor educativa. 
El espacio no tiene por qué limitarse a las paredes que conforman el aula, por el contrario, pueden aprovecharse los corredores, pasillos, explanadas y otros sitios como una extensión del espacio utilizable para la labor educativa.

Por el tipo de actividades que se realizan en el nivel preescolar, y por ende en el Ciclo de Transición, es conveniente que dentro del aula se disponga de una pila de agua y como característica indispensable, se debe considerar una buena ventilación e iluminación natural.

También, como parte del ambiente la decoración es un aspecto muy importante por considerar; asimismo los murales y el material para colocar en las paredes debe ubicarse al alcance de las miradas de los niños. En caso de que este material sea elaborado por el docente, se recomienda prescindir de figuras estereotipadas y de otras culturas, por el contrario es conveniente que para que el aprendizaje sea significativo se utilicen elementos cercanos a la realidad del educando y con pertinencia cultural (Peralta, 1996a). Así, en el caso de Costa Rica, puede utilizarse como referencia, animales del bosque tropical húmedo, del mar, o bien, del entorno más cercano al educando.

No puede dejarse de lado la posibilidad de que las paredes sean decoradas con creaciones artísticas de los niños, o con material traído por ellos.

Por otra parte, el mobiliario debe estar acorde con el currículum que se trabaje; no obstante para efectos de este artículo se recomienda tener estantes de la altura de los niños en cada área para colocar los materiales de trabajo. Asimismo, debe haber un espacio donde los alumnos coloquen sus pertenencias. Con respecto de la cantidad de mesas y sillas, ésta variará dependiendo del espacio con que se cuente, o según las necesidades metodológicas. Es necesario que los niños puedan desplazarse libremente por el aula, así como disponer de suficiente espacio para realizar actividades grupales.

Se recomienda que el aula esté dividida en áreas de trabajo, en las que se coloquen materiales y estímulos que inviten al niño a elegir, explorar, experimentar, clasificar, probar, compartir e interactuar en forma directa con sus iguales y el docente. También, favorecer en el educando la independencia, la autonomía, la responsabilidad, el autocontrol, la cooperación, la concentración, el trabajo en equipo y la organización. 
Estas áreas de trabajo deben estar claramente delimitadas para que el niño pueda distinguir fácilmente el espacio de cada una, sin que se vean como compartimentos aislados. La clara delimitación ayuda a la organización más definida de lo que el niño espera construir en ese espacio. (Zabalza,1996)

Si el espacio disponible lo permite, las áreas de trabajo deben ubicarse alrededor del perímetro del aula, dejando un espacio para desplazarse de una área a otra y para las reuniones grupales. Por el tipo de actividades que se realizan en cada una de las áreas, es importante que aquellas en las que por su naturaleza hay más ruido se ubiquen una al lado de la otra y donde suele haber mayor silencio y concentración se ubiquen en otro sector del aula. De este modo las áreas de dramatización y construcción, estarán separadas de las de artes plásticas y juegos tranquilos. Se recomienda ubicar en las afueras del aula las áreas de agua, arena y carpintería, para evitar derrames de líquidos y arena así como el exceso de ruido dentro del salón de clase.

La cantidad de áreas varía de acuerdo con el espacio físico que se cuente, el planteamiento curricular que se siga y a la riqueza del material que se posea. De esta forma, el arreglo de los espacios se interrelacionan con otros elementos del contexto didáctico. Sin embargo, entendiendo las necesidades de los niños deben ubicarse, al menos, cuatro áreas básicas: dramatización, construcción, juegos tranquilos, y artes plásticas. Si el espacio lo permite, pueden colocarse áreas como el centro de lenguaje, científica, agua, arena, carpintería, entre otras, el espacio exterior también puede ser aprovechado y ubicar allí algunas áreas.

El área de dramatización es de vital importancia, pues por la edad de los niños el juego simbólico representa una de sus actividades principales (Zamora 1996), además de que se le brinda la oportunidad de acercarse a elementos familiares. En consecuencia, debe tener espacio suficiente como para que el niño pueda asumir diferentes papeles que ha observado y experimentado, como el de mamá, papá, bebé, bombero, dependiente, dentista, mascota; o bien, cocinar, llamar por teléfono, hacer una fiesta, ir de compras, entre otros. El dramatizar situaciones o asumir roles de adulto ayuda a los pequeños a encontrarle sentido al mundo de los mayores. También, les da la posibilidad de trabajar en grupo junto a sus compañeros, expresar sentimientos e ideas y utilizar el lenguaje para comunicar sus intereses y necesidades. 
No todos los niños asisten al área de dramatización para asumir roles, algunos lo utilizan para explorar, imaginarse cosas, utilizar herramientas, utensilios o disfrazarse. (Hohmann y otros, 1984)

Para equipar el área de dramatización debe tomarse en cuenta las experiencias previas de los niños, dado que los papeles que asuman son aquellos que han visto en su vida cotidiana, por esto es importante que los implementos sean objetos traídos de la casa por los mismos niños.

Entre los materiales que podrían ubicarse en el área de dramatización se encuentran, cocina, refrigeradora, fregadero de tamaño infantil, ollas de tamaño normal, utensilios de cocina de tamaño normal, vajilla de tamaño normal (platos, vasos, cucharas, platones, bandejas), aplanchador, plancha, escoba, gancho para limpiar, muñeca, cama para muñecas, teléfono, relojes viejos, ropa (vestidos, zapatos para mujer y para hombre, carteras, sacos, sombreros, corbatas, chalecos), joyería (collares, pulsera, aretes, prendedores), disfraces, también es muy importante una mesa con sus sillas que simulen el comedor de la casa.

En el área de construcción es de gran atractivo para los pequeños, probablemente porque la construcción es una de las manifestaciones más antiguas y existe tanto en el niño como en el adulto un impulso interno y una necesidad de construir. En cada construcción que se realiza hay experiencias, elección y organización de los elementos. (Szulanski 1997)

Esta área debe contar con suficiente espacio y estar bien equipada, uno de los materiales primordiales aquí son los bloques de diferente material, que le permitan al niño planear diferentes proyectos y lograr distintos objetivos. Estos bloques le ayudan al niño a formar estructuras verticales, horizontales o circulares, y favorecen las relaciones espaciales, de peso, de tamaño, el equilibrio y permiten crear patrones en las estructuras, trabajar en equipo, clasificar, comparar y ordenar. Por las características de esta área es necesario que cuente con suficiente espacio para que el niño construya libremente, y cuando se caigan, éstas no golpeen a otras personas del salón de clase.

Los bloques deben ser grandes y livianos para que los niños puedan manipularlos libremente y a la vez debe haber otros materiales que motiven la creación de diferentes estructuras, como parques, casas, barrios, edificios, ciudades, granjas, tuberías, túneles, parqueos, carreteras, gasolineras y otros. 
El área de construcción requiere bloques, de diferentes tamaños de $60 \mathrm{~cm} \times 30 \mathrm{~cm}$, hasta de $10 \mathrm{~cm} \mathrm{X} 10 \mathrm{~cm}$, para facilitar el uso. Además cilindros grandes, carros, camiones, bagonetas, tractores, barcos, señales de tránsito, aviones, poleas, establos, animales variados, carretillos, entre otros.

El área de artes plásticas, permite a los niños explorar y experimentar con distintos materiales, aprender cómo funcionan y lo que pueden hacer con ellos. Dado que su principal interés está centrado en el proceso de experimentación, más que los resultados, el trabajo en esta área también favorece la creatividad de la expresión plástica.

Algunos niños familiarizados con los materiales que se les ofrecen en este espacio, pueden usarlos para hacer creaciones, dibujos, collages, figuras con plasticina o arcilla y luego describirlos o crear pequeñas historias.

Es recomendable que esta área se ubique cerca de una pileta y que el piso sea fácil de limpiar, dado que pueden haber derrames de pintura, agua u otros materiales. También, se recomienda tener un tendedero para colocar los trabajos que requieren ser secados antes de guardar, y dejar un espacio para la exposición de las obras realizadas por los pequeños.

Algunos materiales que se recomiendan para el área de plástica son, crayolas, lápices de grafito y de color, témperas, tijeras (para zurdos y derechos), pinceles de diferentes grosores, distintas clases de papel, plasticina, arcilla, perforadores, marcadores, palillos, grapadora, goma, aserrín, esponjas, estarcidos, material de deshecho, prensas para guindar los trabajos, gabachas o delantales para los niños.

Otra de las áreas básicas es la denominada juegos tranquilos, es importante tener claro que este nombre no implica que los niños que trabajen en ella se encuentren en estado pasivo, probablemente su actividad corporal sea más quieta que la de los pequeños que se ubican en dramatización o construcción, no obstante, su actividad mental es totalmente activa; por otro lado, es difícil que donde se encuentre un grupo de niños haya silencio.

El tipo de material que se encuentra en esta área favorece la coordinación fina, acercarse a los libros, escuchar cuentos, armar, desarmar, clasificar y comparar. 
El ambiente de esta área debe ser acogedor y sugestivo, por eso es necesario equiparla con almohadones, una alfombra grande, que permita actuar sobre ella rompecabezas, dominoes, jugar lotería, realizar juegos en grupos pequeños, también poseer estantes a la altura de los niños en los que se ubique el material.

El área de juegos tranquilos generalmente cuenta con libros, rompecabezas, legos, ensartes, loterías, analogías, dominoes, exhibidores para libros, juegos de granja, bloques lógicos y títeres.

El centro de lenguaje es un área que debe estar presente en las aulas preescolares principalmente en el ciclo de transición. Esto se debe a que el ambiente letrado en el que se desarrollan actualmente los preescolares es generalmente muy rico. Son muchos los niños en especial los de zonas más céntricas y urbanas, que están familiarizados con letras y palabras que ven en su ambiente físico, por la observación de letras en los empaques de los alimentos que ingieren diariamente, en la calle, en el supermercado, periódicos, revistas, libros que tienen a su alcance, y otros.

Los pequeños, y más aún los del Ciclo de Transición, por sus características, muestran gran interés por leer y escribir, por esto es necesario brindarles la oportunidad en el centro infantil que lo puedan realizar libremente. En esta área el rol del educador es muy importante dado que no se trata de aplicar un método de lecto-escritura, sino más bien de guiar y estimular los intereses y necesidades que los niños tengan al respecto.

Algunos de los materiales recomendados para esta área son, tarjetas con los nombres de todos los niños del aula, etiquetas en español de productos alimenticios familiares para el niño, palabras que ellos deseen conocer, moldes de letras, letras de diferente textura, crayolas, lápices de color, lápices de grafito, hojas blancas, papel construcción, tijeras (para zurdos y derechos), goma, engrapadora y otros materiales que se consideren pertinentes y por supuesto los de interés para el grupo.

El área de agua por las características de la misma, es muy atractiva para los pequeños, dado que este es uno de los elementos básicos de la naturaleza que, afecta al ser humano desde antes de su nacimiento. Es un elemento indispensable para la existencia del hombre, para 
trabajar, para la limpieza, para disfrutarla y para sus necesidades vitales. Es probable que por las características que posee este elemento, la mayoría de los niños se sientan atraídos a las actividades acuáticas. (Szulanski 1996)

La mesa de agua debe ser de una altura aproximada de $58 \mathrm{cms}$, se recomienda ubicarse de tal forma que los pequeños puedan colocarse en los cuatro lados y tener a su alcance los materiales de trabajo.

Para el trabajo del niño en esta área se recomiendan los siguientes materiales; embudos, mangueras de distintos tamaños y grosores, esponjas de diferentes consistencias, palanganas, baldes, coladores, jeringas, recipientes de tamaños variados, objetos que floten y otros que no, colorante vegetal, champú, gabachas plásticas para que los niños no se mojen su ropa y una toalla.

Con respecto del área de arena es importante resaltar que éste no debe confundirse con el arenero del patio. La arena como elemento natural tiene propiedades diversas: fluida y sólida; cuando está seca se puede utilizar como el agua y cuando está húmeda de otras formas. Por las posibilidades que ofrece, este es uno de los sectores más visitados por los niños, se le puede trabajar con las manos o con utensilios diferentes, permite actividades tridimensionales como construcción y excavación, y bidimensionales, en forma de dibujos (Szulanski 1997). También da la posibilidad de mezclar, remover, amontonar, vaciar, escabar, llenar, verter, golpear, cernir y modelar; se puede experimentar texturas, cantidades (Hohmann, et. al. 1984).

Con respecto al área científica, ésta contribuye a solventar la necesidad que posee el niño de experimentar con diferentes materiales y dar respuesta a preguntas que se plantea. Por otro lado, le ofrece al educando la posibilidad de acercarse a la naturaleza, así que deben incluirse elementos que ofrezcan múltiples opciones para la interpretación, la experimentación y manipulación, la observación y la reflexión sobre temas de su interés.

Algunos materiales que pueden ubicarse en esta área son; una mesa adecuada a la estatura de los niños para que realicen allí experimentos, así como material traído por ellos por ejemplo piedras, hojas, plantas, insectos, también harina, café, sal, azúcar, maicena, colorante vegetal, agua, recipientes para mezclar, envases plásticos, cucharas, lupas, microscopio, y otros elementos necesarios cuando se realiza un experimento específico. 


\section{Mobiliario}

Un espacio abierto con mobiliario disperso, sin una estructura clara da lugar no sólo a una dinámica de trabajo diferente, sino también a una distinta relación de la maestra con sus alumnos, ya sea que trabaje con todos los niños a la vez o en pequeños subgrupos.

Debe tenerse presente que es importante que el mobiliario se encuentre limpio y estéticamente presentable. Este punto está relacionado con la organización de los espacios y el ambiente en el salón de clase. Los estantes como las sillas y las mesas deben estar pintados de forma atractiva para los pequeños, así como también ser livianos para que los puedan transportar libremente.

\section{Materiales}

Los materiales didácticos son un elemento fundamental en el ambiente del aula preescolar, estos provocan la actividad y construcción de conocimiento en el niño y ofrecen una idea del tipo de trabajo que se lleva a cabo en el aula. Por ejemplo, al observar una tina de arena, coladores, embudos, palas, baldes o la pileta de agua con recipientes de diferentes tamaños, mangueras y vasos, se puede deducir las actividades que los niños llevan a cabo en dichos espacios o áreas.

Así también los materiales pueden facilitar interacciones, tener diferentes objetivos, motivar diversas actividades y fomentar la creatividad, entre otros.

Los materiales comerciales, los recuperables del medio, o los traídos de la casa tienen implícitos valores y una determinada concepción metodológica.

Los materiales de desecho por ejemplo, embaces, cajas o botellas de plástico y de cartón, o los del entorno como semillas, piedras, hojas, palillos, entre otros, favorecen la educación ambiental y educación para el consumo, y también fomentan la creatividad al buscar nuevas opciones en el uso de material que fue concebido para otro fin.

Es conveniente que los materiales ofrezcan al niño diferentes alternativas para su uso, de esta forma se está fomentando la creatividad e indirectamente el pequeño interioriza que ante un conflicto existen diferentes posibilidades para la resolución de problemas. 


\section{Conclusiones}

Desde el nacimiento de la educación preescolar el ambiente ha jugado un papel importante, por esto sus propulsores hicieron referencia a él. También, investigadores como Piaget y Vigotski resaltaron en sus trabajos la forma en que este elemento contribuye en el aprendizaje y en el desarrollo de los niños.

Las interacciones sociales que lleve a cabo el educando en el aula, con el docente y compañeros así como el medio que le rodea, son componentes fundamentales en la construcción del conocimiento.

La organización del aula es tan importante que la disposición de los elementos, indica el tipo de interrelaciones que allí se dan, el planteamiento o concepción metodológica que posee el maestro, el papel que juegan el docente y el niño, entre otros.

Es necesario que la decoración y el material de trabajo sean cercanos a la realidad del niño, que posean pertinencia cultural, lo que contribuye a favorecer su aprendizaje significativo.

Dado que los niños pasan gran parte de la rutina diaria dentro del salón de clase, es conveniente que el docente tenga presente algunas características fundamentales que debe poseer el aula. Es importante dividirla en áreas, espacios o sectores, porque esto contribuye a que el niño se organice mentalmente, sea más fácil la elección y planificación de su trabajo. Es conveniente dejar el espacio necesario para que los pequeños puedan desplazarse fácilmente por el salón de clase y sus relaciones interpersonales no se afecten.

Considerando las características y necesidades de los niños de 6 años las áreas básicas que se recomienda tener en el aula son: dramatización, dado que en ella se pueden reproducir situaciones cercanas a las vividas por los niños en su vida cotidiana; construcción, por ser ésta una de las manifestaciones propias del ser humano; juegos tranquilos, que favorece la concentración en actividades lógico matemático, y el acercamiento a la literatura, y el área de artes plásticas en la que el niño tiene la posibilidad de experimentar y crear libremente diversos diseños.

Si el espacio dentro del aula o fuera de ella lo permite puede aumentarse el número de áreas, como la de agua, la de arena o la de carpintería, las que por sus características son de 
gran placer para los pequeños; también, puede colocarse el área científica que contribuye a solventar la necesidad de experimentación, y teniendo en cuenta las experiencias previas que poseen los niños relacionadas con ambientes letrados, es conveniente el centro de lenguaje.

Las áreas deben equiparse con material que permita la interacción de los niños con los compañeros, el maestro y los mismos elementos que las componen, para favorecer la construcción del conocimiento.

Con respecto de los materiales, estos pueden ser de diferentes procedencias, por ejemplo: comerciales, recuperables del medio o de desecho.

Si bien es cierto, el ambiente del salón de clase es básico para el adecuado desarrollo integral del niño, no puede dejarse de lado la importancia que tiene el educador como parte fundamental de este proceso, ya que es él quien favorece o da la oportunidad al educando de interactuar con el ambiente escolar, y de esta manera propiciar la construcción de su conocimiento al interactuar con el medio.

\section{REFERENCIAS}

García Lizano, Nidia, et al. (1992). Conocimiento, participación y cambio espacio en el aula. San José, Costa Rica: Editorial de la Universidad de Costa Rica.

Hohmann, Mary, Banet, Bernard, Weikart David. (1984). Niños Pequeños en Acción. México: Trillas.

Iglesias, Lina. (1996). La organización de los espacios en la educación infantil. En Zabala, Miguel, Calidad en la educación infantil. Madrid, España: Ediciones Narcea.

Kamii, Constance, Lewis, Barbara, Mellizo, Felipe. (1997). Contribución de Jean Piaget a la educación infantil. Revista Educar de 0 a 6 años. Enero febrero.

Montessori, María. (1939). Manual práctico del método. Barcelona, España: Araluce.

Peralta, Victoria. (1996a). El currículo en el jardín de infantes (Un análisis crítico). Santiago de Chile: Editorial Andrés Bello.

Peralta, Victoria. (1996b). Currículos educacionales en América Latina. Su pertinencia cultural. Santiago de Chile: Editorial Andrés Bello.

Szulanki, Susana, Stein Rebeca. (1997). Educación Preescolar en Israel. Una experiencia significativa. Tel Aviv, Israel: Editorial Airora. 
Vila, Ignacia. (1997). Vigotski y la educación infantil. Revista Educar de 0 a 6 años. Madrid España. Julio agosto.

Zamora, Marisela. (1998). Educación Científica y Matemática para el niño preescolar I. San José Costa Rica: Editorial EUNED. 